\title{
EFECTO DE LA CAL EN LA ESTABILIZACIÓN DE SUBRASANTES
}

Fabián Elizondo Arrieta

Alejandro Navas Carro

Denia Sibaja Obando

\section{Resumen}

Por las características geológicas de Costa Rica, los suelos de subrasante presentes son en su mayoría suelos con altos índices de plasticidad. Estas subrasantes son utilizadas como cimiento para la estructura de pavimentos o en rutas no pavimentas (tierra y lastre) en superficies que estarán en contacto directo con las cargas del tránsito en su estado natural o mezcladas con materiales granulares en los casos de caminos de lastre.

Consecuentemente se plantea el uso de la cal como un estabilizador ideal para materiales finos de media o alta plasticidad, el cual no ha sido utilizado en Costa Rica para estos fines. Su uso tiene como consecuencia una serie de mejoras tales como: el aumento de propiedades mecánicas de resistencia, menor susceptibilidad a la humedad, reducción de la plasticidad y aumento de trabajabilidad, entre otros.

Por lo anterior se planteó el siguiente proyecto enfocado en evaluar el efecto de dos tipos de cal (primera y segunda calidad) en las propiedades de un suelo de alta plasticidad, estabilizado con cal y valorar el cumplimiento de especificaciones de acuerdo con la normativa existente.

Palabras claves: subrasantes, cal, estabilización de subrasantes.

\begin{abstract}
Because of the geology of Costa Rica, sub grade soils are mostly high plasticity. These are used as the foundation sub-grades for pavement structures or as a surface course material in unpaved roads in which case, it will be in direct contact with traffic loads, either as sub-grade roads or mixed with granular materials in gravel roads.

Lime is recognized as an ideal stabilizer for fine materials of medium and high plasticity, which has not been used extensively for this purpose in Costa Rica. Many improvements are related with the use of lime in fine materials such as increased mechanical resistance; reduce moisture susceptibility, reduction of plasticity and increase workability, etc.

Therefore we proposed this project, focused on evaluating the effect of two types of lime (first and second quality) on the properties of a high plasticity soil and assess compliance of specifications in accordance with existing regulations.
\end{abstract}

Keywords: subgrade, lime, stabilization of subgrade.

Recibido: 23 de noviembre de 2009 Aprobado: 12 de abril de 2010.

\section{INTRODUCCIÓN}

Por las características geológicas de Costa Rica, los suelos de subrasante presentes en gran parte del país, son suelos con altos índices de plasticidad. Estas subrasantes son utilizadas en pavimentos como cimiento para su estructura o en rutas no pavimentas (tierra y lastre) como superficies que estarán en contacto directo con las cargas del tránsito, ya sea en su estado natural o mezcladas con materiales granulares en los casos de caminos de lastre.

Las características de alta plasticidad están relacionadas con baja capacidad mecánica, gran susceptibilidad a la humedad y el consecuente efecto en la consistencia o deformación plástica ante las cargas.

Como antecedente a este proyecto durante el año 2008 se presenta por parte de la Unidad de Investigación en Infraestructura Vial del 
LanammeUCR, un proyecto de investigación enfocado en recopilar información y experiencias en el tema de la estabilización y mejoramiento de rutas no pavimentadas, teniendo como producto la Guía para la estabilización y mejoramiento de rutas no pavimentadas.

Esta guía presenta el uso de la cal como un estabilizador ideal para materiales finos de media o alta plasticidad. Puesto que el uso de la cal tiene como consecuencia una serie de mejoras tales como: el aumento de propiedades mecánicas de resistencia, reducción de la plasticidad, aumento de la trabajabilidad, entre otros. Adicionalmente la guía presenta un procedimiento para el diseño y evaluación del desempeño en los laboratorios de materiales estabilizados con cal que requiere ser implementado y valorar su aplicabilidad en Costa Rica.

La estabilización de suelos con cal ha sido utilizada en otros países con gran éxito, logrando aumentar la vida útil de rutas no pavimentadas, disminuyendo la frecuencia en el mantenimiento que al mediano y largo plazo resultan ser soluciones competitivamente económicas, ofreciendo mejores superficies de ruedo y que indiscutiblemente presentan un mejor desempeño ante condiciones de lluvia y humedad extrema, tal y como las condiciones presentes en Costa Rica, en donde muchas zonas del país poseen una época lluviosa que abarca más del $50 \%$ del año.

Es importante agregar que este tipo de soluciones, no son exclusivas de carreteras de lastre, también son utilizadas en el mejoramiento de sub-rasantes dentro de una estructura de pavimento para volúmenes medios y altos de tránsito, mejorando por mucho la capacidad estructural de estos materiales, impermeabilizando el resto de capas de la estructura y evitando la migración de finos o contaminación del paquete estructural con la consecuente desmejora en su desempeño.

El objetivo general de esta investigación fue la valoración del efecto de la cal disponible en Costa Rica, utilizada como aditivo estabilizador de materiales finos que presenten índices de plasticidad (IP) medios y altos, y su efecto en las propiedades mecánicas y de durabilidad del material estabilizado.

\section{CONCEPTOS GENERALES Y ESQUEMA DE LA INVESTIGACIÓN}

\subsection{Factores que se deben de considerar}

Los factores que deben de ser considerados en la selección de la cal como estabilizador adecuado para un suelo son: el tipo de suelo a estabilizar, el propósito para el cual la capa será utilizada, el tipo de mejora que se desea del suelo (objetivo de la estabilización), la resistencia requerida y durabilidad de la capa estabilizada, las condiciones ambientales y el costo.

Existen algunas consideraciones generales que permiten seleccionar a la cal como el estabilizador más apto para un suelo específico; estas son la granulometría y la plasticidad o textura del suelo. Como regla general la cal reaccionará con los suelos de mediana y alta plasticidad produciendo un decremento en el Índice de Plasticidad, aumento de trabajabilidad, disminución de expansión, y aumento de resistencia. La cal es utilizada para la estabilización de una gran variedad de materiales incluyendo suelos de muy baja resistencia, transformándolos en una superficie de trabajo estable o con propiedades mecánicas similares a las de una sub-base (material granular); además con cantidades marginales de materiales granulares como las grava-arcillas, conocidas también como gravas sucias pueden formar una base de alta resistencia y calidad. En la práctica es común la utilización de pequeñas cantidades de cemento Portland junto con la cal para aumentar resistencia.

\subsection{Propiedades requeridas de un suelo para ser estabilizado con cal}

Como procedimiento general se incluyen en este apartado una metodología para seleccionar aditivos aptos para estabilizar un suelo, incluyendo los suelos recomendados para ser estabilizados con cal. En la Figura 1 se presenta una categorización del tipo de suelo basado en las características granulométricas del suelo, específicamente en el tamaño de las 


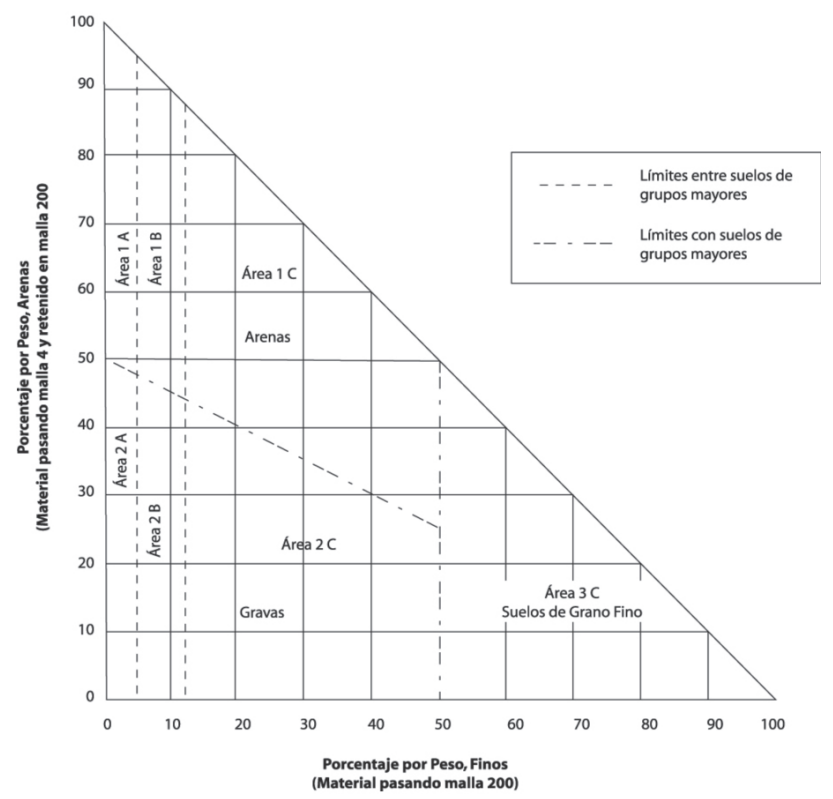

Figura 1. Selección de los aditivos.

Fuente: (Department of The Army, The Navy, and The Air Force, 1994).

partículas y porcentajes retenidos en la malla $\mathrm{N}^{\circ} 4(4,75 \mathrm{~mm})$ y la malla $\mathrm{N}^{\circ} 200(75 \mu \mathrm{m})$. Este triángulo es dividido en varias áreas de suelos con tamaños de partículas similares y características de pulverización.

El proceso de selección continúa con el Cuadro 1, en la cual se especifican para cada área de suelos mostrados en la Figura 1, opciones de aditivos y restricciones basados en el tamaño de partícula o condiciones de plasticidad (IP, LL).

En la segunda columna del Cuadro 1 se muestra una lista con los tipos de suelos (SUCS) que entrarían dentro de esta categoría, lo cual se utilizaría para comprobar que el área fue seleccionada correctamente.

El proceso de selección de las opciones de aditivo requiere de la información granulométrica y los límites de Atterberg del suelo. La información requerida para ingresar en la Figura 1 es: el porcentaje de material pasando la malla $\mathrm{N}^{\circ}$ $200(75 \mu \mathrm{m})$ y el porcentaje de material pasando la malla $\mathrm{N}^{\circ} 4(4,75 \mathrm{~mm})$ pero retenido en la malla $\mathrm{N}^{\mathrm{o}} 200(75 \mu \mathrm{m})$. Se ingresa a la Figura 1 con estos dos valores y el área correspondiente (1 A, 2A, 3, etc.) es ubicada mediante la intersección de estos dos valores.
El área determinada mediante la Figura 1 es ubicada en la primera columna de la Cuadro 1 y el tipo de suelo podrá ser chequeado en la segunda columna. Las opciones de aditivos para la estabilización según el tipo de suelo son propuestos en la tercer columna y las restricciones para el uso de cada aditivo (incluyendo la cal) son presentadas en las columnas siguientes; restricciones establecidas para prevenir el uso de algunos aditivos estabilizadores bajo condiciones particulares del suelo, para las cuales no son aplicables.

Dentro de la investigación se analizaron dos metodologías de diseño y se evaluó el desempeño con el fin de valorar las ventajas y desventajas que cada una de ellas, se presenta a continuación un resumen de las metodologías utilizadas.

2.3. Metodología de diseño: Cuerpo de Ingenieros de la Armada Norteamericana (CI) (Department of The Army, The Navy, and The Air Force, 1994)

- Paso 1: Cálculo del contenido inicial de cal mediante la valoración del $\mathrm{pH}$ de varias 
Cuadro 1. Matriz para la selección del aditivo estabilizador.

\section{Área Clase de suelo}

\section{Restricción de LL e IP del suelo}

\section{Tipo de estabilizador} aditivo recomendado
SW ó SP

(1) Bituminosos

(2) Cemento Pórtland

(3) Cal-cemento-ceniza volante

$1 \mathrm{~B}$
SW-SM o
(1) Bituminosos
SP-SM o
(2) Cemento Pórtland
SW-SC o
(3) $\mathrm{Cal}$
SP-SC
(4) Cal-cemento-ceniza volante
SM o SC
(1) Bituminosos
o SM-SC
(2) Cemento Pórtland
(3) $\mathrm{Cal}$
(4) Cal-cemento-ceniza volante

$1 \mathrm{C}$
GW o GP

(1) Bituminosos

(2) Cemento Pórtland

2B
GW-GM o
(1) Bituminosos
GP-GM o
(2) Cemento Pórtland
GW-GC o
(3) $\mathrm{Cal}$
GP-GC
(4) Cal-cemento-ceniza volante

2C

$\begin{array}{ll}\text { GM o GC } & \text { (1) Bituminosos } \\ \text { o GM-GC } & \text { (2) Cemento Pórtland } \\ & \text { (3) Cal } \\ & \text { (4) Cal-cemento-ceniza volante }\end{array}$

(4) Cal-cemento-ceniza volante
(3) Cal-cemento-ceniza volante

IP no exceda a 10

IP no exceda a 30

IP no exceda a 12

IP no exceda a 25

IP no exceda a 10

IP no menor a 12

IP no exceda a 25

IP no exceda a 25

IP no exceda a 10

IP no exceda a 30

IP no menor de 12

IP no exceda a 25

IP no exceda a 10

IP no menor de 12

IP no exceda a 25

LL menor de $40 \mathrm{e}$

IP menor de 10

o $\mathrm{MH} \mathrm{o}$

$\mathrm{ML}$ o $\mathrm{OH}$

o OL o

(2) $\mathrm{Cal}$

ML-CL

\section{Restricción en \\ porcentaje pasando}

la malla No. 200

\section{Comentarios}

No exceda el $30 \%$ por peso

Material bien graduado.

Debe contener al menos un $45 \%$ por peso del material pasando la malla No. 4

Material bien graduado.

Debe contener al menos un $45 \%$ por peso del material pasando la malla No. 4

No exceda $30 \%$ por peso

Material bien graduado solamente
Materiales orgánicos y altamente ácidos que se encuentran dentro de esta área no son susceptibles a la estabilización por mecanismos ordinarios.

a La clasificación del suelo corresponde a MIL-STD-619B. Restricción en el límite líquido y el índice de plasticidad (IP) está de acuerdo con el Método 103 en MIL-STD-621A.

$\mathbf{b}_{\text {IP }} \leq 20+50$ - porcentaje pasando malla No. $200(75 \mu \mathrm{m})$ 
muestras de suelo cal preparadas a diferentes niveles o dosificaciones $(2 \%, 4 \%, 6 \%, 8 \%)$. El contenido de cal más bajo al cual se logra un $\mathrm{pH}$ de alrededor de 12,4 será el contenido estimado inicial de cal.

- Paso 2: Utilizando el contenido inicial de cal, se deben llevar a cabo ensayos de humedad densidad para determinar la densidad máxima y el contenido de humedad óptima. El ensayo de densidad humedad deberá realizarse de acuerdo con la norma ASTM D 1557 (Próctor Modificado).

- Paso 3: Preparar muestras por triplicado de la mezcla suelo cal, para los ensayos de compresión inconfinada y durabilidad. Los contenidos de cal a los cuales se deben de preparar las mezclas serán: el contenido de cal determinado en el paso $2 \mathrm{y}$, al $2 \%$ y al $4 \%$ de cal por encima del contenido de cal determinado en el paso 2. Los especimenes preparados se deberán de curar por un periodo de $28 \mathrm{~d}$ a $23{ }^{\circ} \mathrm{C}$. También se puede utilizar un curado acelerado por $48 \mathrm{~h}$ a $49^{\circ} \mathrm{C}$.

- Paso 4: Tres especimenes se deberán de fallar a compresión inconfinada de acuerdo con el procedimiento ASTM D1633, y otros tres especimenes deberán ser sometidos al ensayo de durabilidad conforme a la norma ASTM D559

- Paso 5: Se deberán de comparar los resultados obtenidos con los requerimientos solicitados de acuerdo con las especificaciones contractuales, o con las especificaciones recomendadas en los Cuadros 2 y 3 . El contenido de cal más bajo, el cual cumpla con los requerimientos para los resultados de compresión inconfinada y demuestre la durabilidad requerida será el contenido de cal de diseño.

Cuadro 2. Resistencia mínima a la compresión inconfinada para suelos estabilizados

Capa de suelo estabilizada

Base

Sub base, material selecto y subrasante
Resistencia mínima a la compresión $(\mathbf{k P a})$

Pavimento Flexible Pavimento Rígido

5170

3450

1725

1380

Resistencia mínima a la compresión determinada a los veintiocho días para la estabilización con cal.

Fuente: (Adaptado de Department of The Army, The Navy, and The Air Force, 1994).

Cuadro 3. Requerimientos de durabilidad

Capa de suelo estabilizada

Granular IP $<10$
Granular IP $>10$
Limos

Arcillas
Pérdida máxima permitida luego de doce ciclos de humedecido y secado o ciclos de congelamiento y descongelamiento, como el porcentaje de peso inicial del espécimen 
2.4. Metodología de diseño: National Lime Association (NLA). (National Lime Association, 2004)

La National Lime Association de los Estados Unidos evaluó varios procedimientos para el diseño de mezclas estabilizadas con cal con el fin de generar uno definitivo que pudiera ser utilizado con confianza para diversos tipos de suelo y climas.

- Paso 1: Evaluar las propiedades claves del suelo como un paso inicial para determinar si el suelo es adecuado para a ser estabilizado con cal.

- Paso 2: Determinación de la demanda estimada inicial de cal, el porcentaje más bajo de cal en el suelo que alcanza un $\mathrm{pH}$ en laboratorio de 12,4 es el porcentaje mínimo de cal para estabilizar un suelo.

- Paso 3: Determinación del contenido de humedad óptimo y la máxima densidad seca del suelo tratado con cal, mediante el procedimiento ASTM D698 (Próctor Estándar).

- Paso 4: Fabricación de los especímenes para compresión inconfinada (CI) mediante el procedimiento ASTM D5102 procedimiento B. La muestra debe de ser almacenada en una bolsa sellada e impermeable por $1 \mathrm{~h}-24 \mathrm{~h}$ antes de fabricar el espécimen de ensayo.

- Paso 5: Curado y confinamiento de los especímenes de compresión inconfinada (CI). Inmediatamente después de la fabricación de los especímenes, deben de ser envueltos en

Cuadro 4. Recomendaciones para resistencias a compresión inconfinada de mezclas de suelo-limoso.

\begin{tabular}{|c|c|c|c|c|}
\hline \multirow{2}{*}{ Uso previsto } & \multirow{2}{*}{$\begin{array}{c}\text { Saturación por } \\
\mathbf{8 d}\end{array}$} & \multicolumn{3}{|c|}{ Ciclos congelación-descongelación a } \\
\hline & & tres ciclos & siete ciclos & diez ciclos \\
\hline & $(\mathrm{kPa})$ & $(\mathrm{kPa})$ & $(\mathrm{kPa})$ & $(\mathrm{kPa})$ \\
\hline \multicolumn{5}{|l|}{ SubBase } \\
\hline Pavimento rígido/Losas & 345 & 345 & 621 & 827 \\
\hline $\begin{array}{l}\text { Pavimento flexible } \\
\qquad(>25 \mathrm{~cm})^{b}\end{array}$ & 414 & 414 & 689 & 896 \\
\hline $\begin{array}{l}\text { Pavimento flexible } \\
(20 \mathrm{~cm}-25 \mathrm{~cm})^{b}\end{array}$ & 483 & 483 & 689 & 965 \\
\hline $\begin{array}{l}\text { Pavimento flexible } \\
(12,5 \mathrm{~cm}-20 \mathrm{~cm})^{\mathrm{b}}\end{array}$ & 621 & 621 & 896 & 1103 \\
\hline \multicolumn{5}{|l|}{ Base } \\
\hline & 896 & 896 & 1172 & 1379 \\
\hline
\end{tabular}


plástico y sellados en una bolsa impermeable. Los especímenes deben ser sometidos a un periodo de curado por $7 \mathrm{~d} \mathrm{a} 40{ }^{\circ} \mathrm{C}$. Someter los especímenes por $24 \mathrm{~h}$ a un remojo por capilaridad.

- Paso 6: Determinación de la compresión inconfinada (CI) de los especímenes curados y condicionados en humedad. Utilizar el procedimiento ASTM D5102 B para determinar la CI de los especímenes curados y condicionados en humedad. La CI es el promedio de al menos dos especímenes. En el Cuadro 3 se presentan valores sugeridos para CI.

\section{RESULTADOS OBTENIDOS}

Cuando se realizan ensayos para el diseño de dosificaciones de un material, en el laboratorio se intentan representar las condiciones presentes en el proyecto, los resultados obtenidos bajo estas condiciones deberán ser comparados con especificaciones las cuales garanticen el comportamiento adecuado de este material. En el caso de suelos estabilizados con cal la definición de especificaciones se realiza de manera empírica, es decir, mediante la comparación de resultados obtenidos en el laboratorio y el desempeño del material en campo. Con base en lo anterior es posible indicar que existe un vínculo entre el método de diseño propuesto y las especificaciones asociadas, así como con los procesos constructivos implementados.

Dentro de los objetivos de este proyecto se plantea la valoración de dos métodos de diseño, cada uno con sus propias especificaciones y que responden a las condiciones para las cuales fue calibrado empíricamente bajo las condiciones que intenta representar.

Las características del suelo utilizado en este estudio se muestra en el Cuadro 5, el cual puede caracterizarse conforme al Sistema Unificado de Clasificación de Suelos, SUCS, como un Limo de Alta Plasticidad (IP=25) y con un CBR del 6,6 \%, escogido por considerarse como un material crítico, de baja resistencia y alta susceptibilidad a la deformación.

Con la finalidad de valorar si la cal que se produce en Costa Rica produce una estabilización que logre cumplir con los requerimientos solicitados, se analizaron dos

Cuadro 5. Caracterización del suelo utilizado en el estudio.

$\begin{array}{ccc} & \text { Porcentaje de finos } & 85 \% \\ \text { Granulometría } & \text { Porcentaje de gravas } & 1 \% \\ & \text { Porcentaje de arenas } & 14 \% \\ & \text { Porcentaje de arcillas } & 65 \% \\ \text { Límites de Atterberg } & \text { LL } & 63 \\ \text { Próctor } & \text { LP } & 38 \\ \text { Estándar y CBR } & \text { IP } & 25 \\ \text { Clasificación } & \gamma d \text { max (kg/m3) } & 32,98 \\ & \text { CBR } & 1376,7 \\ & \text { SUCS } & 6,6 \\ & \text { AASHTO } & \text { A- } 7-5\end{array}$

Fuente: (Los autores, 2009). 
tipos de cal definidos como: cal de primera y cal de segunda. La caracterización de la cal se realizó mediante parámetros de: óxido de calcio, óxido de magnesio, dióxido de carbono, porcentaje de humedad, nivel de acidez y aspectos de granulometría. Se entiende como cal de primera, aquella que presenta mayores contenidos de óxido de calcio y óxido de magnesio, además de presentar una granulometría más fina, lo que se traduce en una cal de mayor de calidad. Al mismo tiempo, se tomaron muestras en diferentes épocas del año con la finalidad de evaluar la variabilidad en el tiempo de las propiedades de la cal debido al proceso de producción de la misma, las cuales se denominaron como Muestra 1, Muestra 2 y
Muestra 3. Como se puede ver en los Cuadros 6 y 7 existe un incumplimiento de sus propiedades respecto a las especificaciones requeridas para su uso en estabilización, en el parámetro correspondiente al contenido de óxido de calcio y óxido de magnesio, estos componentes son los encargados de reaccionar con los materiales plásticos del suelo para producir un efecto cementante por lo que entre menores sean sus proporciones se esperarían mayores contenidos de cal para lograr la estabilización.

Otra característica de las muestras de cal analizadas es la alta variabilidad que inclusive provoca incumplimientos en otras propiedades, ambas situaciones se pueden observar en la Figuras 2 y 3.

Cuadro 6. Caracterización y variabilidad de la cal (Cal de primera).

\begin{tabular}{lcccc}
\multicolumn{1}{c}{ Parámetro } & Muestra 1 & Muestra 2 & Muestra 3 & Especificación \\
Óxido de calcio (\% masa/masa) & $70,8 \pm 0,5$ & $56,4 \pm 0,5$ & $87,1 \pm 0,2$ & \\
Óxido de magnesio $(\%$ masa/masa) & $0,5 \pm 0,1$ & $1,36 \pm 0,2$ & $3,8 \pm 0,1$ & $90 \%$ mínimo \\
Dióxido de carbono $(\%$ masa/masa) & $3,0 \pm 0,3$ & $4,0 \pm 0,2$ & $5,6 \pm 0,1$ & $5 \%$ máximo \\
Porcentaje de humedad $(\%)$ & $1,6 \pm 0,1$ & $0,79 \pm 0,05$ & $0,10 \pm 0,08$ & $2 \%$ máximo \\
Malla 30 $(600 \mu \mathrm{m})$ & $0,35 \pm 0,07$ & $0,15 \pm 0,07$ & $23,1 \pm 0,4$ & $3 \%$ máximo \\
Malla 200 $(75 \mu \mathrm{m})$ & $12,1 \pm 0,4$ & $8,2 \pm 0,4$ & $9,9 \pm 0,5$ & $25 \%$ máximo \\
Charola & $87,8 \pm 0,6$ & $91,9 \pm 0,3$ & $88,6 \pm 0,1$ &
\end{tabular}

Fuente: (Los autores, 2009).

Cuadro 7. Caracterización y variabilidad de la cal (Cal de segunda).

\begin{tabular}{lcccc}
\multicolumn{1}{c}{ Parámetro } & Muestra 1 & Muestra 2 & Muestra 3 & Especificación \\
Óxido de calcio (\% masa/masa) & $43,0 \pm 0,5$ & $44,6 \pm 0,5$ & $68,2 \pm 0,2$ & \\
Óxido de magnesio (\% masa/masa) & $0,6 \pm 0,1$ & $1,37 \pm 0,5$ & $4,6 \pm 0,1$ & mínimo \\
Dióxido de carbono (\% masa/masa) & $3,6 \pm 0,3$ & $3,7 \pm 0,2$ & $20,6 \pm 0,1$ & $5 \%$ máximo \\
Porcentaje de humedad $(\%)$ & $0,91 \pm 0,05$ & $0,77 \pm 0,07$ & $0,09 \pm 0,05$ & $2 \%$ máximo \\
Malla 30 $(600 \mu \mathrm{m})$ & $2,9 \pm 2$ & $23,1 \pm 0,4$ & $49,2 \pm 0,6$ & $3 \%$ máximo \\
Malla $200(75 \mu \mathrm{m})$ & $24,8 \pm 0,2$ & $11,0 \pm 0,6$ & $35,8 \pm 0,5$ & $25 \%$ máximo \\
Charola & $46 \pm 1$ & $66,1 \pm 0,1$ & $15,2 \pm 0,1$ &
\end{tabular}




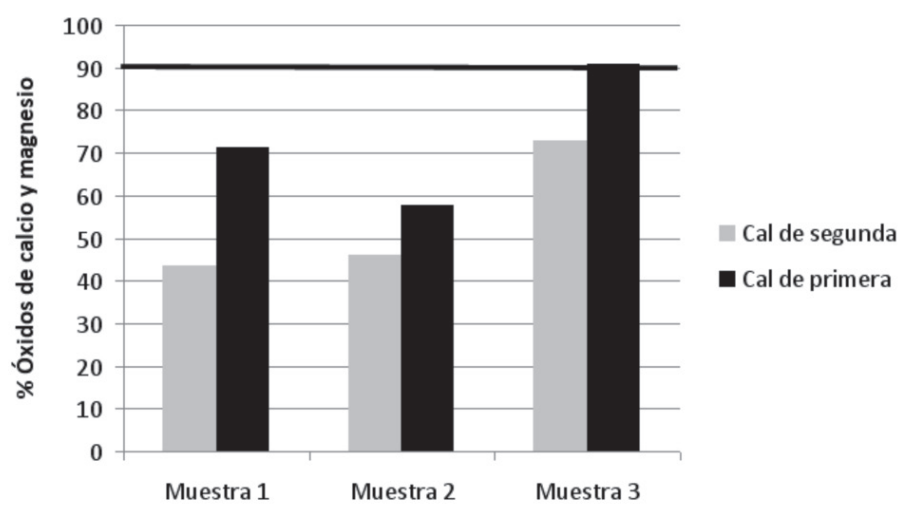

Figura 2. Óxidos de calcio y de magnesio (\%)

Fuente: (Los autores, 2009).

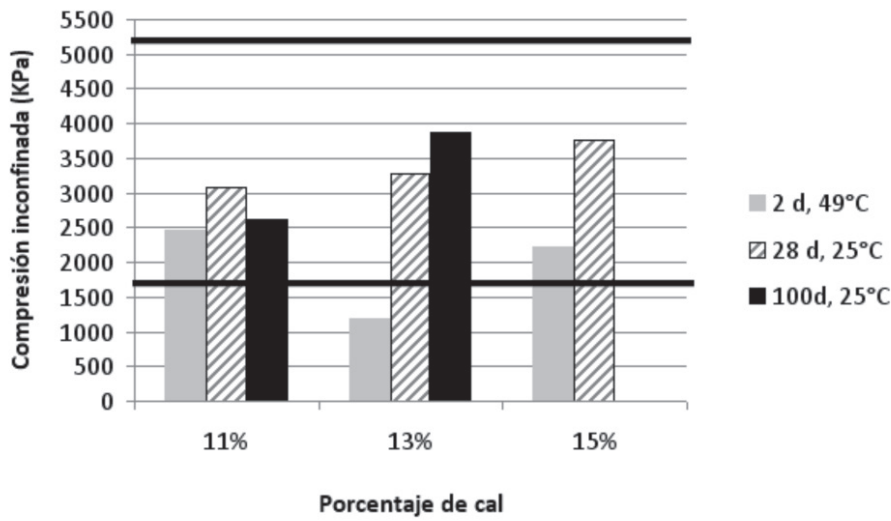

Figura 3. Compresión inconfinada (CI)

Fuente: (Los autores, 2009).

Ambos métodos de diseño establecen un cálculo estimado inicial de cal mediante la medición del $\mathrm{pH}$ del suelo, los resultados obtenidos en el estudio definen un $4 \%$ para la cal de primera y un $6 \%$ para la cal de segunda aproximadamente (Cuadro 8). No obstante para la valoración del Método de Diseño del CI, se analizó únicamente la cal de primera, contenidos de cal correspondientes al $11 \%$, al $13 \%$ y al $15 \%$, y los dos condicionamientos propuestos por el método: $28 \mathrm{~d}$ a $25^{\circ} \mathrm{C}$ y $48 \mathrm{~h}$ a $49{ }^{\circ} \mathrm{C}$.

El método del Cuerpo de Ingenieros de la Armada Norteamericana (CI) propone el cumplimiento de dos requerimientos para las subrasantes estabilizadas con cal (Cuadros 2 y 3) las cuales corresponden a la compresión inconfinada y la durabilidad mediante ciclos de humedecido y secado.

De los resultados mostrados en Cuadro 9, los cuales corresponden a El método del Cuerpo de Ingenieros de la Armada Norteamericana se puede comentar lo siguiente: en cuanto al requisito de resistencia a la compresión inconfinada los especimenes curados por un periodo de 2 d a $49{ }^{\circ} \mathrm{C}$ presentan una alta 
variabilidad y no muestran una tendencia a aumentar su resistencia conforme se incrementa el contenido de cal (Figura 3). Los especimenes curados por un periodo de $28 \mathrm{~d}$ a $25^{\circ} \mathrm{C}$ tienden a ser menos variables e incrementan su resistencia conforme aumenta los contenidos de cal.

Al comparar los resultados con las especificaciones propuestas en el método se puede identificar que los tres porcentajes utilizados se encuentran por encima de la resistencia a la compresión mínima (1725 kPa) solicitada para utilizar el material como sub base o material selecto en pavimentos flexibles, inclusive los resultados están muy por encima de este valor, con resistencias que van desde los $3103 \mathrm{kPa}$ hasta los $3790 \mathrm{kPa}$ aproximadamente lo que hace pensar que las dosificaciones pueden ser reducidas y aun así cumplirán con los requisitos de resistencia, sin embargo no llegan a ser suficiente para ser utilizada como una base.
En cuanto al ensayo de durabilidad los resultados obtenidos para las tres dosificaciones se encuentran por debajo del máximo especificado para limos, correspondiente al $8 \%$. Los valores de módulo resiliente o capacidad mecánica, pese a que no son requeridos por el método de diseño se presentan con el fin de evidenciar que se encuentran cercanos a los $200 \mathrm{MPa}$, los cuales corresponden a valores similares a los que se obtendría con una base granular.

Una de las limitantes más importantes del método del Cuerpo de Ingenieros de la Armada Norteamericana es el tiempo que toma la realización del diseño, considerando que el condicionamiento acelerado de los especimenes $\left(48 \mathrm{~h}\right.$ a $\left.49{ }^{\circ} \mathrm{C}\right)$ no logró ser representativo del curado normal de la cal $\left(28 \mathrm{~d} \mathrm{a} 25^{\circ} \mathrm{C}\right)$ el tiempo total que tarda la realización del diseño corresponde a un poco más de dos meses, tomando en cuenta que la preparación de los especimenes

Cuadro 8. Cálculo del contenido estimado inicial de cal mediante $\mathrm{pH}$.

Cal de primera (Muestra 1)

Masa

\begin{tabular}{|c|c|c|c|c|c|c|c|c|c|}
\hline Beaker & Cal & $\begin{array}{c}\text { de } \\
\text { agua }\end{array}$ & $\begin{array}{l}\text { Masa } \\
\text { de cal }\end{array}$ & $\mathbf{p H}$ & Temperatura & $\begin{array}{l}\text { Masa } \\
\text { agua }\end{array}$ & $\begin{array}{c}\text { Masa de } \\
\text { cal }\end{array}$ & pH & Temperatura \\
\hline & $(\%)$ & (g) & (g) & & $\left({ }^{\circ} \mathrm{C}\right)$ & (g) & (g) & & $\left({ }^{\circ} \mathrm{C}\right)$ \\
\hline 1 & 1 & 100 & 0,310 & 10,67 & 24,8 & 100 & 0,310 & 10,53 & 24,2 \\
\hline 2 & 2 & 100 & 0,620 & 12,02 & 24,9 & 100 & 0,620 & 11,63 & 24,3 \\
\hline 3 & 3 & 100 & 0,929 & 12,46 & 25,2 & 100 & 0,929 & 12,07 & 24,1 \\
\hline 4 & 4 & 100 & 1,239 & 12,66 & 24,5 & 100 & 1,239 & 12,38 & 24,2 \\
\hline 5 & 5 & 100 & 1,549 & 12,67 & 24,7 & 100 & 1,549 & 12,59 & 24,1 \\
\hline 6 & 6 & 100 & 1,859 & 12,68 & 24,6 & 100 & 1,859 & 12,75 & 24,1 \\
\hline 7 & 7 & 100 & 2,168 & 12,72 & 24,4 & 100 & 2,168 & 12,72 & 24,3 \\
\hline 8 & 8 & 100 & 2,479 & 12,73 & 24,4 & 100 & 2,479 & 12,75 & 24,2 \\
\hline 9 & 9 & 100 & 2,788 & 12,73 & 24,5 & - & - & - & - \\
\hline 10 & 10 & 100 & 3,098 & 12,74 & 24,0 & - & - & - & - \\
\hline 11 & 11 & 100 & 3,407 & 12,74 & 24,3 & - & - & - & - \\
\hline 12 & 12 & 100 & 3,717 & 12,72 & 24,1 & - & - & - & - \\
\hline 13 & 13 & 100 & 4,026 & 12,73 & 24,0 & - & - & - & - \\
\hline
\end{tabular}

Cal de segunda (Muestra 2)

Fuente: (Los autores, 2009). 
Cuadro 9. Resultados de ensayos de desempeño. Cal de primera. Método de diseño del cuerpo de Ingenieros de los Estados Unidos.

\begin{tabular}{|c|c|c|c|c|c|}
\hline \multirow{3}{*}{ Porcentaje de $c$} & \multicolumn{3}{|r|}{$\begin{array}{l}\text { Pérdidas } \\
\text { Suelo-Cal }\end{array}$} & \multicolumn{2}{|c|}{ Modulo resiliente } \\
\hline & \multicolumn{2}{|c|}{$(\mathrm{kPa})$} & $(\%)$ & \multicolumn{2}{|c|}{ (MPa) } \\
\hline & $2 \mathrm{~d} 49^{\circ} \mathrm{C}$ & $28 \mathrm{~d} 25^{\circ} \mathrm{C}$ & & Máximo & Mínimo \\
\hline \multirow{3}{*}{$11 \%$} & 2167 & 3393 & 5,56 & 161,02 & 114,02 \\
\hline & 1971 & 2981 & 4,30 & 181,60 & 128,78 \\
\hline & 3285 & 2893 & - & 172,08 & 125,69 \\
\hline \multirow[t]{2}{*}{ Promedio } & 2475 & 3089 & 4,93 & 171,56 & 122,83 \\
\hline & 1451 & 3815 & 6,83 & 219,36 & 118,63 \\
\hline \multirow[t]{2}{*}{$13 \%$} & 971 & 2295 & 8,03 & 136,25 & 65,39 \\
\hline & 1128 & 3707 & - & 270,20 & 166,11 \\
\hline \multirow[t]{2}{*}{ Promedio } & 1183 & 3272 & 7,43 & 208,60 & 116,71 \\
\hline & 1912 & 3913 & 4,94 & 143,27 & 104,09 \\
\hline \multirow[t]{2}{*}{$15 \%$} & 2020 & 3609 & 6,70 & 198,34 & 93,58 \\
\hline & 2756 & 3776 & - & 247,11 & 198,81 \\
\hline Promedio & 2229 & 3766 & 5,82 & 196,24 & 132,24 \\
\hline
\end{tabular}

Fuente: (Los autores, 2009).

tarda una semana, el condicionamiento 28 d, más el tiempo que se demora hacer los doce ciclos del ensayo de durabilidad que corresponden a otros $28 \mathrm{~d}$, hacen del método poco práctico para utilizarse en el campo. Es por esta razón que se planteó el diseño mediante el método de la NLA, este método tiene la ventaja que elimina el ensayo de durabilidad y lo sustituye por variaciones en las especificaciones de compresión inconfinada de acuerdo con las condiciones climáticas al que el suelo estabilizado estará sometido (Cuadro 4), además modifica el proceso de condicionamiento $\left(7 \mathrm{~d}\right.$ a $\left.40^{\circ} \mathrm{C}\right)$ el cual requiere que los especimenes se introduzcan en bolsas selladas que permitan mantener la humedad dentro del espécimen, factor clave para lograr la optimización de la reacción con la cal.

Una condición a tomar en cuenta es que los especímenes deben de ser compactados mediante Próctor estándar y no modificado como se especifica en el método del CI.

Bajo el esquema anterior se realizaron los ensayos para los dos tipos de cal, las dosificaciones utilizadas fueron las estimadas mediante el método del pH más $2 \%$ y más $4 \%$ en cada caso, además como parte del estudio de investigación se evaluó el ensayo de durabilidad y módulo resiliente. Sin embargo para el primero, los especimenes no resistieron los ciclos de humedecido y secado, no obstante se debe recordar que este ensayo no forma parte de este procedimiento de diseño y no debería relacionarse con un posible mal desempeño del material ya que como se mencionó anteriormente existe una correlación entre los métodos de diseño y calibraciones que se han hecho con desempeños en campo, por lo que al existir diferencias en el método de compactación en laboratorio no podría especificarse un requisito para estas condiciones a menos que exista una validación. Los resultados obtenidos se presentan en el Cuadro 10 y en la Figura 4 se sintetizan las tendencias.

Como se puede observar en el Cuadro 10, existen algunas diferencias en los resultados de compresión inconfinada para los diferentes contenidos de cal. Si se tiene en consideración 


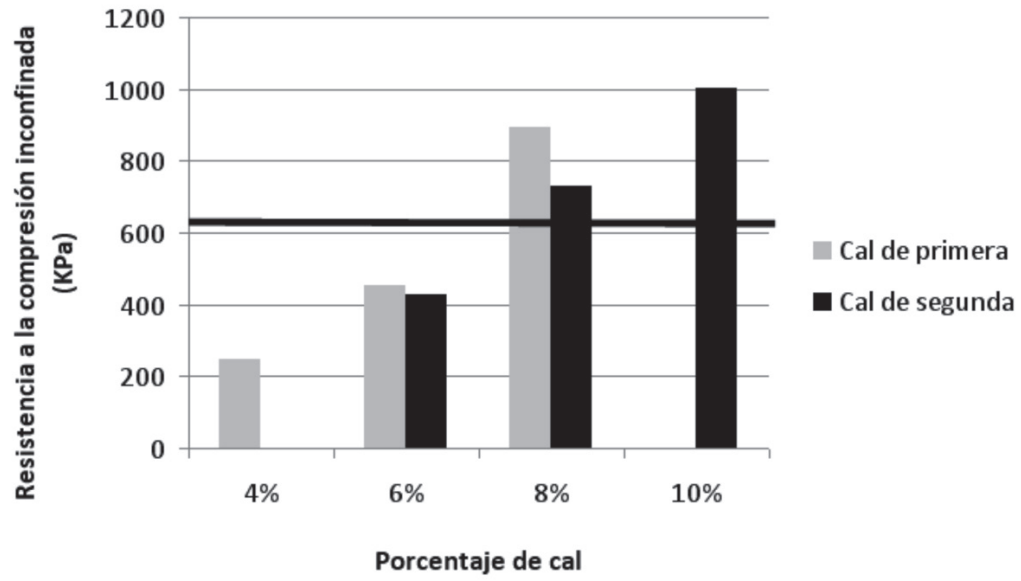

Figura 4. Compresión inconfinada (NLA) Fuente: (Los autores, 2009).

Cuadro 10. Resultados de ensayos de desempeño. Cal de primera y segunda. Método de diseño del Lime Association.

\begin{tabular}{|c|c|c|c|c|c|c|}
\hline \multirow{5}{*}{ Porcentaje de cal } & \multicolumn{2}{|c|}{ Compresión inconfinada } & \multicolumn{2}{|c|}{ Módulo resiliente } & \multicolumn{2}{|c|}{ Módulo resiliente } \\
\hline & \multicolumn{2}{|c|}{$7 \mathrm{~d}$ y $40^{\circ} \mathrm{C}$} & \multicolumn{2}{|c|}{ Cal de primera } & \multicolumn{2}{|c|}{ Cal de segunda } \\
\hline & \multicolumn{2}{|c|}{$(\mathrm{kPa})$} & \multicolumn{2}{|c|}{$(\mathrm{MPa})$} & \multicolumn{2}{|c|}{ (MPa) } \\
\hline & Cal de & Cal de & & & & \\
\hline & & & Máximo & Mínimo & Máximo & Mínimo \\
\hline & & & & & & \\
\hline & 200 & - & $15, / 1$ & 36,34 & $\begin{array}{lll}- & - \\
\end{array}$ & - \\
\hline \multirow[t]{2}{*}{$4 \%$} & 265 & - & 113,69 & 82,42 & - & - \\
\hline & 275 & - & 72,47 & 39,20 & - & - \\
\hline \multirow[t]{2}{*}{ Promedio } & 248 & - & 87,29 & 52.65 & - & - \\
\hline & 471 & 441 & 139,50 & 64,78 & 131,6 & 88,40 \\
\hline \multirow[t]{2}{*}{$6 \%$} & 422 & 481 & 123,30 & 82,85 & 78,91 & 49,11 \\
\hline & 471 & 363 & 72,64 & 46,44 & - & - \\
\hline \multirow[t]{2}{*}{ Promedio } & 454 & 428 & 111,81 & 64,69 & 105,26 & 68,75 \\
\hline & 755 & 785 & 80,98 & 47,50 & 111,32 & 74,10 \\
\hline \multirow[t]{2}{*}{$8 \%$} & 932 & 726 & 187,16 & 137,93 & 96,86 & 67,39 \\
\hline & 991 & 676 & 82,28 & 55,05 & - & - \\
\hline \multirow[t]{2}{*}{ Promedio } & 892 & 729 & 116,81 & 80,16 & 104.09 & 70,74 \\
\hline & - & 980 & - & - & 113,93 & 69,63 \\
\hline \multirow[t]{2}{*}{$10 \%$} & - & 941 & - & - & 211,22 & 124,14 \\
\hline & - & 1089 & - & - & - & - \\
\hline Promedio & & 1004 & - & - & 162,58 & 96,88 \\
\hline
\end{tabular}

Fuente: (Los autores, 2009). 


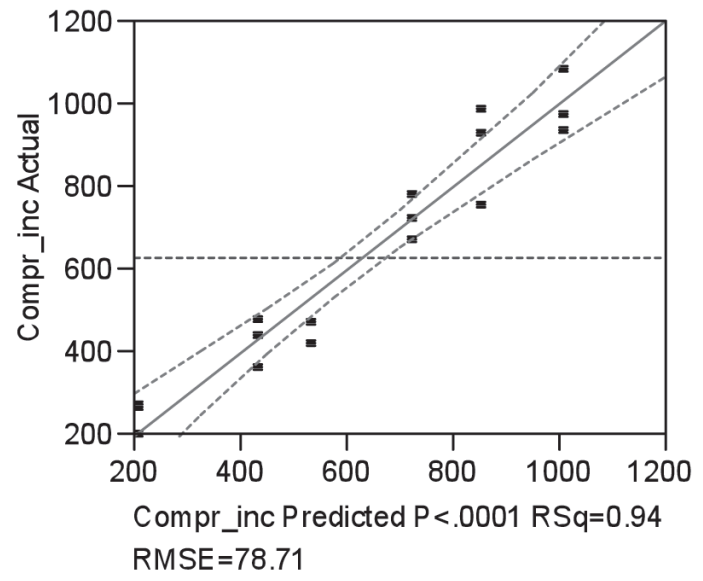

Figura 5. Ajuste del modelo y límites de confianza. Fuente: (Los autores, 2009).

Cuadro 11. Resumen de resultados del modelo estadístico obtenido.

\section{Summary of Fit}

RSquare

RSquare Adj

Root Mean Square Error

Mean of Response

Observations (or Sum Wgts)
0,936421

0,922797

78,71016

626,1111

18

\section{Analysis of Variance}

$\begin{array}{lcccc}\text { Source } & \text { DF } & \text { Sum of squares } & \text { Mean Square } & \text { FRatio } \\ \text { Model } & 3 & 1277465,7 & 425822 & 68,7332 \\ \text { Error } & 14 & 86734,1 & 6195 & \text { Prob }>\text { F } \\ \text { C. } & & & & \\ \text { Total } & 17 & 1364199,8 & & <0,0001\end{array}$


las diferencias existentes en el contenido de óxido de calcio y óxido de magnesio para las muestras de cal denominadas como $\mathrm{Cal}$ de Primera y Cal de Segunda para la primera fecha de muestreo, se realizó un análisis estadístico con el paquete estadístico JMP 4.0, para establecer si las diferencias en los resultados de la resistencia a la compresión inconfinada se debe al efecto de las variaciones en el contenido de cal, en conjunto con el efecto del tipo de cal (cal de primera y cal de segunda), así como el efecto combinado de ambas variaciones. La Figura 5 y el Cuadro 11 muestran los resultados obtenidos, como se puede notar el modelo es altamente significativo puesto que la

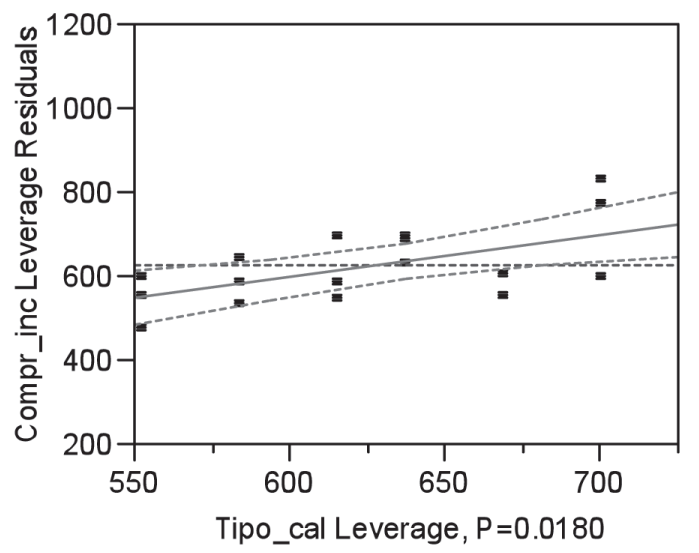

Figura 6. Representación gráfica de la prueba de significancia del efecto del tipo de cal. Fuente: (Los autores, 2009). probabilidad asociada a la $\mathrm{F}$ muestral es $<0,0001$.

Por otra parte, el modelo de análisis lleva implicita la comparación entre la $\mathrm{Cal}$ de Primera y la Cal de Segunda. Esta comparación se hace utilizando contrastes ortogonales, las Figuras 6 y 7 , y el Cuadro 12 muestran los resultados obtenidos, de manera tal que se establece que la resistencia a la compresión inconfinada disminuye cuando se reduce la calidad de la cal. El contraste realizado compara la resistencia a la compresión con Cal de Primera con la de Cal de Segunda, la probabilidad asociada al valor de $t$ es igual a 0,018 por lo que puede afirmarse a un $95 \%$ de

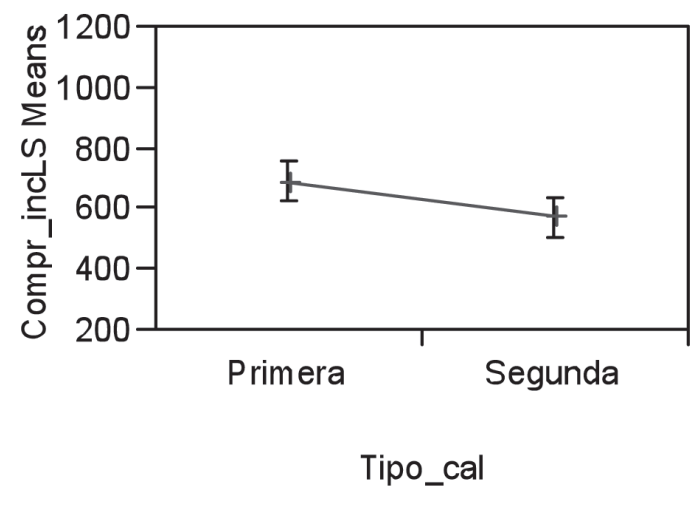

Figura 7. Representación gráfica del comportamiento de la resistencia a la compresión al cambiar el tipo de cal.

Fuente: (Los autores, 2009).

Cuadro 12. Resumen de resultados de la comparación del tipo de cal utilizando contrastes ortogonales.

$\begin{array}{cc}\text { Primera } & 1 \\ \text { Segunda } & -1 \\ \text { Estimate } & 116,53 \\ \text { Std Error } & 43,509 \\ \text { t Ratio } & 2,6783 \\ \text { Prob }>|t| & 0,018 \\ \text { SS } & 44439\end{array}$

Fuente: (Los autores, 2009) 
confianza que existe un mayor efecto o mejoría en resistencias a la compresión inconfinada alcanzadas con la cal de primera respecto a la cal de segunda.

Se realizaron análisis estadísticos similares para evaluar el efecto del tipo de cal y el contenido de cal para los resultados de módulo resiliente, sin embargo los resultados obtenidos no fueron significativos ni concluyentes para los ensayos realizados, por lo que es necesario continuar profundizando en este tema. De esta manera y de acuerdo con los resultados obtenidos y siendo la resistencia inconfinada el único parámetro especificado para valorar el cumplimiento o no de una subrasante estabilizada por este método (Cuadro 4). Por otro lado, para las condiciones existentes en Costa Rica, en las cuales no hay ciclos de congelamiento y descongelamiento, es posible utilizar dosificaciones del $6 \%$ de cal para estabilizar un material que vaya a ser utilizado como sub base en pavimentos con estructuras robustas de más de $25 \mathrm{~cm}$ sobre la capa estabilizada; en caso de estructuras con espesores a los $12 \mathrm{~cm}$, se requerirá entonces dosificaciones cercanas al $8 \%$. Por último si el material estabilizado fuera a utilizarse como una base la dosificación que se debe de emplear deberá ser cercana al $10 \%$.

\section{CONCLUSIONES}

1. De los resultados obtenidos se evidencia la mejoría en las propiedades mecánicas y de durabilidad en el material de subrasante cuanto se incorpora la cal como material estabilizador. Con base en los resultados obtenidos, esta mejoría se incrementa conforme se agrega un mayor porcentaje de contenido de cal. En el caso de los resultados de compresión inconfinada, se observa una mayor mejoría cuando se emplea cal con mayores contenidos de óxido de calcio y óxido de magnesio (propiedades de la cal de primera).

2. Es recomendable profundizar en el análisis y valoración de los efectos de la cal en los valores de módulo resiliente de materiales de subrasante estabilizados y determinar cuáles propiedades tales como el contenido de cal, el proceso de compactación, o bien determinar que otros factores influyen en el desempeño de los mismos.

3. La cal evaluada en el estudio presenta una alta variabilidad en sus propiedades físicoquímicas e incumplimientos respecto a los requisitos requeridos internacionalmente, producto posiblemente del proceso de producción en el momento de muestreo; debido a esto se propone un estudio que busque cuantificar el efecto de esta variabilidad en las propiedades mecánicas del material estabilizado así como analizar otras fuentes de cal para valorar si se repite esta condición.

4. El método de la NLA se propone como un método más factible para ser propuesto como método de diseño debido a la reducción en el tiempo requerido para el diseño y su correspondencia con los resultados del método del CI.

5. Con dosificaciones iguales o mayores al $6 \%$ se pueden lograr materiales que de acuerdo con los procedimientos de diseño y especificación permiten transformar un material de alta plasticidad y muy susceptible a deformarse en un material con propiedades que permita ser utilizado como sub base e inclusive como base.

6. Se recomienda ampliar esta investigación a otros tipos de materiales que presenten condiciones aptas para ser estabilizado con cal, además se hace necesario la construcción de tramos de prueba que permitan valor en campo el desempeño real de material.

\section{REFERENCIAS BIBLIOGRÁFICAS}

Elizondo, F., Sibaja, D. (2008). Guía para la estabilización y mejoramiento de rutas no pavimentadas. Unidad de Investigación en Infraestructura Vial. Laboratorio Nacional de Materiales y Modelos Estructurales. San José, Costa Rica.

Department of The Army, The Navy, and the Air Force (1994). Soil Stabilization for 
pavements. Washington D.C, United States.

National Lime Association, (2004). Manual de estabilización de suelos tratados con cal, United States.

Federal Highway Administration, (1993). Soil and Base Stabilization and Associated Drainage Consideration. Washington D.C, United States.

\section{SOBRE LOS AUTORES}

\section{Fabián Elizondo Arrieta}

Ingeniero civil

Unidad de Investigación en Infraestructura Vial

Laboratorio Nacional de Materiales y Modelos

Estructurales

Universidad de Costa Rica

San José, Costa Rica
Correo electrónico: fabian.elizondo@ucr.ac.cr

\author{
Alejandro Navas Carro \\ Ingeniero civil \\ Unidad de Investigación en Infraestructura Vial \\ Laboratorio Nacional de Materiales y Modelos \\ Estructurales \\ Universidad de Costa Rica \\ San José, Costa Rica \\ Correo electrónico: anavas@lanamme.ucr.ac.cr
}

\section{Denia Sibaja Obando}

Ingeniera civil

Unidad de Investigación en Infraestructura Vial

Laboratorio Nacional de Materiales y Modelos

Estructurales

Universidad de Costa Rica

San José, Costa Rica

Correo electrónico: dsibaja@lanamme.ucr.ac.cr 